\title{
Annalen der Physik
}

(C) Johann Ambrosius Barth 1993

\section{Thermal motion of one-dimensional domain walls in monolayers of a polar polymer observed by Video-STM}

\author{
Ch. Ludwig, G. Eberle, B. Gompf, J. Petersen, and W. Eisenmenger \\ 1. Physikalisches Institut, Universität Stuttgart, Pfaffenwaidring 57, W-7000 Stuttgart 80 , Germany
}

Received 5 February 1993, revised version received 1 March 1993, accepted 3 March 1993

\begin{abstract}
Scanning tunneling microscopy (STM) has been used to investigate monolayers of the ferroelectric copolymer polyvinylidenefluoride/trifluoroethylene $\mathrm{P}$ (VDF/TrFE) showing images of ordered polymer monolayers. By scanning with video frame rate, direct observation of the motion of onedimensional domain walls was also possible for the first time. The images clearly show domain walls normal to the polymer chains. From measurements of the temperature dependence of the domain wall velocities the activation energy for the thermally generated kink motion was estimated. These results are compared with theoretical models describing domain wall motion in ferroelectric PVDF.
\end{abstract}

Keywords: Scanning tunneling microscopy; Ferroelectricity; Domain wall dynamics.

\section{Introduction}

Since the discovery of the strong piezoelectric effect in PVDF [1] and its copolymer with TFFE the properties of these materials have been extensively studied, but the origin of the "hard" ferroelectric behavior of the polymer is still under discussion. In ferromagnetic materials two intrinsic mechanisms exist to stabilize the magnetization in the case of strong anisotropy energy: Either small single domain crystallites with high domain wall nucleation energy resist polarization reversal or the domain walls in multidomain crystals are pinned by defects, hindering domain wall motion. In ferroelectric materials an additional mechanism is possible: the stabilisation of the polarization by charge injection and charge trapping at the surface of polarized crystallites $[2,3]$.

To describe domain wall motion during the poling process in the ferroelectric polymer PVDF two models were discussed in literature both involving kink motion i.e. a $180^{\circ}$ twisting of the macromolecule about its longitudinal axis (see Fig. $1 \mathrm{a}, \mathrm{b}$ ): DveyAharon et al. [4] describe domain wall motion by thermal generation and field enhanced motion of individual kinks along the polymer chains corresponding to domain walls parallel to the chains (Fig. 1a). Pertsev and Zembil'gotov [5] explain the domain wall motion by a collective motion of kinks forming domain walls normal to the polymer chains (Fig. I b).

In the following we show that it is possible to obtain specific information on the microscopic process for the special case of a monolayer of $\mathrm{P}$ (VDF/TrFE) on graphite by scanning tunneling microscopy. STM has been successfully used for studying organic materials, although the contrast mechanism is still under discussion $[6,7,8]$. The local piezoelectric activity of thin $\mathrm{P}(\mathrm{VDF} / \mathrm{TrFE})$ films has been imaged [9] recently. There 
a)

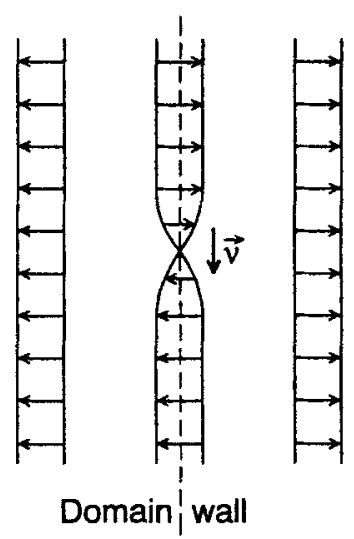

b)

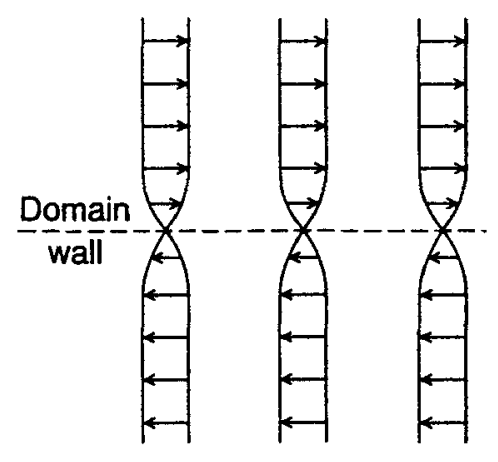

Fig. 1 (a) In the model of Dvey-Aharon et al. [4] under an external field an individual kink runs, after thermal generation at the domain wall, along the polymer chain with sound velocity. The domain walls are parallel to the polymer chains. The domain wall motion under high electric field is determined by the time for creating a kink. (b) In the model by Pertsev [5] a long range interaction leads to a collective motion of the kinks which are present in thermal equilibrium. The domain walls are normal to the polymer chains and are directly driven by an applied field.

exist a few investigations showing molecular dynamics in real time [10] or individual polymer chains [11]. Ferroelectric domain structures have been imaged by scanning [12] and transmission electron microscopy [13] and by force microscopy [14].

\section{Experimental procedures}

The STM investigations were carried out with a specially developed Video-STM producing images in the constant height mode at a video frame rate of 20 frames $/ \mathrm{sec}$. The images are recorded with a video camera from a CRT-monitor and stored on videotape. The whole microscope is temperature stabilized and the temperature can be varied between $77 \mathrm{~K}$ and $300 \mathrm{~K}$. In this way the thermal drift is reduced in operation below $1 \AA / s$ in the whole temperature range. The STM images were obtained in a nitrogen atmosphere with Pt-Ir tips. All images presented in this paper represent unfiltered raw data directly photographed from a TV-monitor without any further computer-processing. On graphite it is easy to prepare clean and flat surfaces but it has the disadvantage of weak interaction with adsorbates. Therefore we dried off a drop of trichlorobenzene on the graphite surface before cleaving it. Only with graphite pretreated in this way it was possible to prepare $\mathrm{P}(\mathrm{VDF} / \mathrm{TrFE}) 75: 25 \mathrm{~mol} \%$ monolayers from solution (solvent: methylethylketon). It is presently unknown what specific mechanism is responsible for this pretreatment influence. Neither the pretreated graphite nor the pretreated graphite with solvent only show any structure different from clean graphite in the STM image. $\mathrm{X}$-ray analysis showed that intercalation can be excluded.

\section{Results and conclusions}

Fig. 2 a shows a Video-STM image of a monolayer of the copolymer P(VDF-TrFE) at room temperature prepared in the way described before. With $I=1 \mathrm{nA}$ and $U=600 \mathrm{mV}$ 


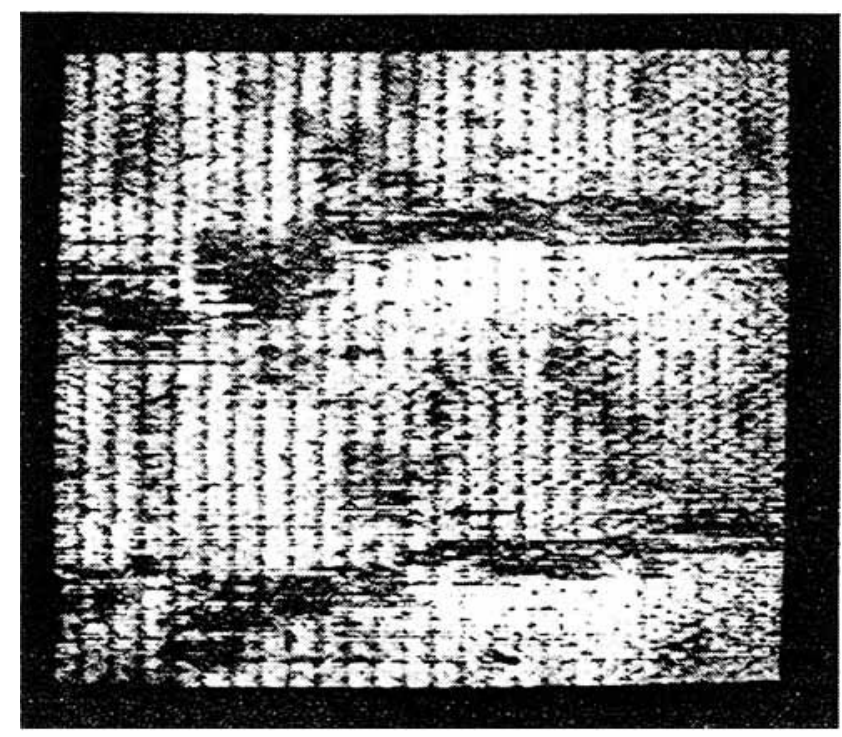

Fig. 2 (a) Video-STM image of a monolayer of $\mathrm{P}$ (VDF/TeFE) on graphite at room temperature. The incividual polymer chains are ciearly resolved. The dark narrow zones perpendicular to the chains are domain walls which fluctuate thermaily in their position wille the chains stay in place. (b) Same Video scan $200 \mathrm{~ms}$ later. Image area: $100 \AA \times 100 \AA$, scanning speed: 5 frames/s. Thermal drift of the inage less than $\AA$.

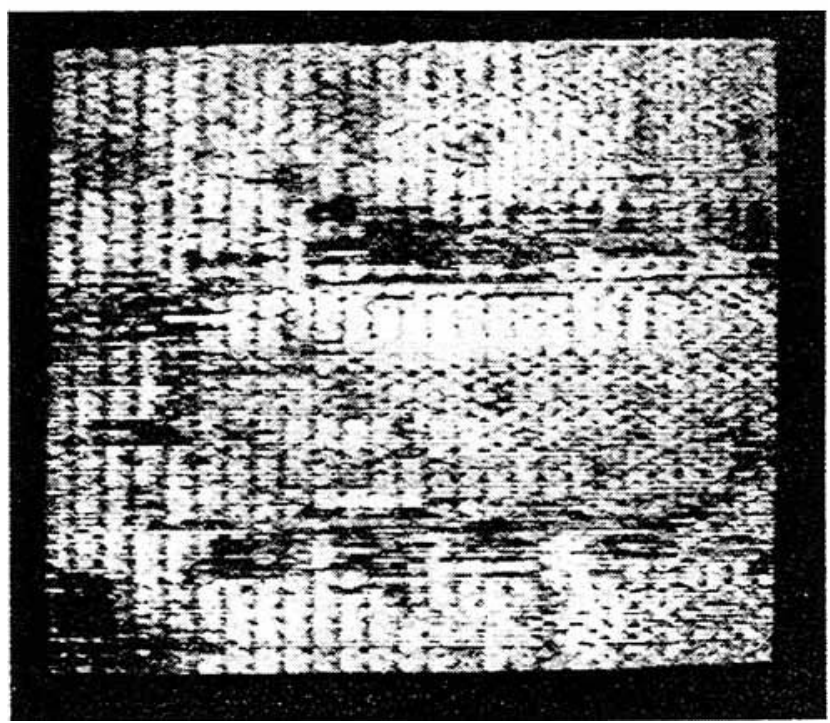

tunneling current and voltage, respectively, we achieved the best quality of the current images. The polymer chains are clearly resolved as bright granular stripes and thin dark lines. They form two-dimensional polymer crystallites with a distance of about $4.3 \AA$ between adjacent chains, which corresponds to the distance between second next carbon rows of the graphite surface ( $4.26 \AA$ ). The expected thickness of a monolayer PVDF is about $3 \AA$, but the observed step hight in the constant current mode at the filmsubstrate edge was always less than $3 \AA$. Therefore we are sure that we observe only monolayers. By decreasing the tunneling resistance the underlying graphite becomes visible. In this way it is possible to determine the alignment of the polymer chains with 
respect to the graphite lattice. The polymer chains are always parallel to one of the three symmetry equivalent lattice vectors and in registry with every second carbon row. There is no superstructure observed in direction of the chains, which means that the monomer units $(5.12 \AA)$ of the polymer are in full registry with the underlaying graphite $(2 \times 2.46 \AA)$. The polymer chain structure of Fig. $2 \mathrm{a}$ is, however, interrupted by irregular narrow dark zones normal to the chain direction. The position of these zones moves statistically in the sequence of frames as can be seen by Fig. $2 \mathrm{~b}$ from the same video scan as Fig. 2 a but $200 \mathrm{~ms}$ later. Motion due to thermal drift would be less than $1 \AA$ in this short time period and can therefore be neglected. Note also that the chain position is not altered. We interprete these narrow dark zones as domain walls between two $180^{\circ}$ polarisation directions. In the more common slow scan constant current mode the domain walls are completely washed out.

Fig. 3 shows in addition a grain boundary between two crystallites of different chain directions at room temperature In $\mathrm{P}(\mathrm{VDF} / \mathrm{TrFE})$ the chain length is about $10^{4}$ monomers which corresponds to a molecular weight of about $5 \times 10^{5}$ [15]. At this length it is unlikely that all polymer chains end at the grain boundary. Therefore we expect that the chains are folded there. This is confirmed by the observed thermal motion of the grain boundary at room temperature with immediate recrystallisation and a small amorphous regime between adjacent grains.

With our Video STM we are able to observe the motion of the grain boundaries of Fig. 3 as well as of the domain walls in Fig. 2 in real time. At room temperature the motions are observed on a time scale of about $100 \mathrm{~ms}$, the time resolution of our STM.

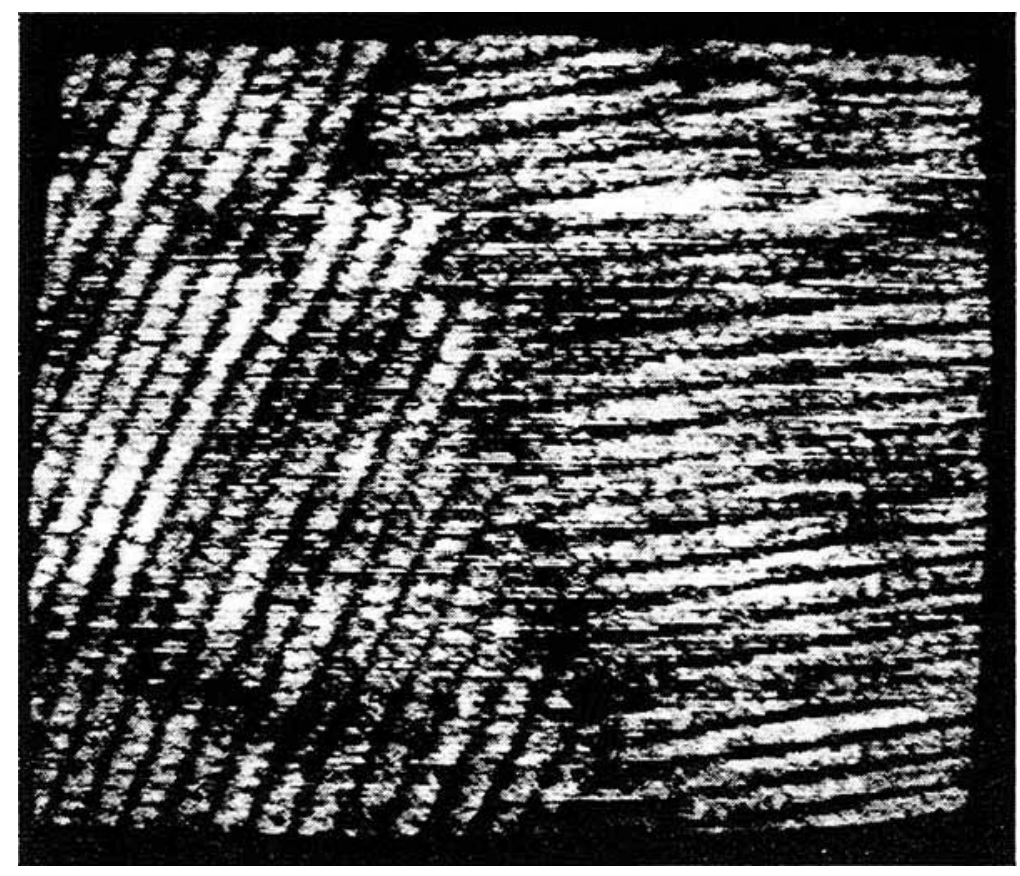

Fig. 3 Grain bouncary between two polymer crystailites. The chains are always parallei to the main graphite directions. At room temperature we see the fluctuations of these grain bouncaries in real time. Image size: $100 \dot{A} \times 100 \dot{A}$. 
The images do not change during electronic rotation of the scanning direction, which means that the fluctuations are not included by the scanning process itself. Reduction of the temperature to $225 \mathrm{~K}$ leads to a slowing down of the domain wall motion. To quantify the temperature dependence of the domain wall motion we determined the average displacement of a domain wall between two subsequent frames and multiplied it with the frame rate in the temperature range between $300 \mathrm{~K}$ and $225 \mathrm{~K}$. Below $225 \mathrm{~K}$ the average domain wall velocity is less than $1 \AA$ /s, the drift limit of our microscope, above $300 \mathrm{~K}$ the domain wall motions are larger than $20 \mathrm{~nm} / \mathrm{s}$ and this is too fast for our Video-STM. The resulting preliminary average speed was plotted in an Arrhenius diagram as shown in Fig. 4. As expected for thermally activated motion, the experimental velocity data are in agreement with an exponential dependence on temperature with an activation energy of about $200-300 \mathrm{meV}$.

Fig. 4 Arrhenius plot of average domain wall velocity versus inverse ternperature. As expected for thermally activated motion, our preliminary experimental data are in agreement with an exponential dependence on temperature corresponding to an activation energy of $200-300 \mathrm{meV}$.

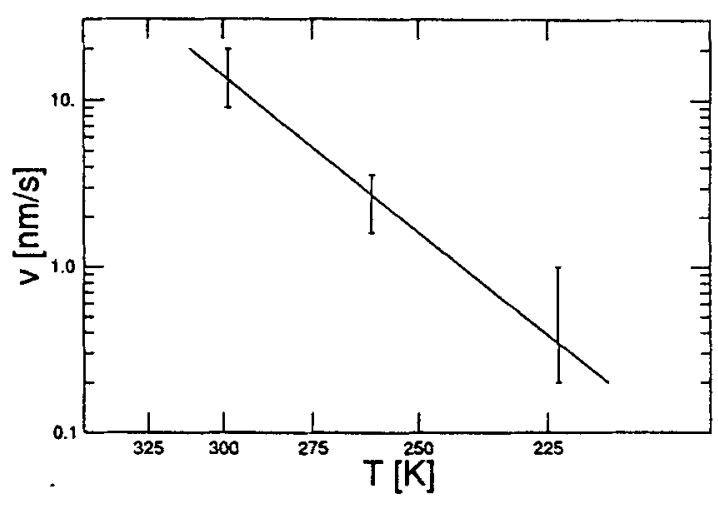

In the polar $\beta$-phase of the copolymer the dipole moment per monomer is about $5 \times 10^{-30} \mathrm{~cm}$. Simple energy consideration leads us to the conclusion that due to the induced mirror charges in the conductive surface the orientation of the polymer chains is probably with their dipole moments parallel to the surface plane. An orientation perpendicular to the surface would require an energy of about $10 \mathrm{eV}$ per monomer. From X-ray diffraction data it is known that in the bulk copolymer the distance between adjacent chains parallel to the dipole orientation is about $4.9 \AA$ [16]. In the monolayer we observed $4.3 \AA$. That means that the strong dipole moment in combination with a conducting substrate leeds to a lateral compression of the monolayer by more than $13 \%$ compared to the bulk structure.

In $\mathrm{P}$ (VDF/TrFE) we have a non-polar C-atom-skeleton with external strongly polar $\mathrm{H}$ - and F-sidegroups. When the dipoles are oriented parallel to the surface plane we get an alternating structure of high and low polarizability (see Fig. 5). In the C-atom skeleton between the graphite substrate and the tunneling tip we expect a small polarizability, whereas the adjacent $F$ and $H$ ligands of neighboring molecules extending between substrate and tunneling tip provide an increased electronic polarizability. In the regime of high polarizability the barrier height is probably reduced [17]. Therefore in Fig. 2 the thin dark lines are in our view the polymer skeleton and the bright granular stripes are the polar sidegroups of two adjacent chains. This is consistent with the observation in Fig. 2 a that the dark domain wall zones end half way within the bright stripes (see left part of Fig. 2 a). The strong reduction of the tunneling prob- 


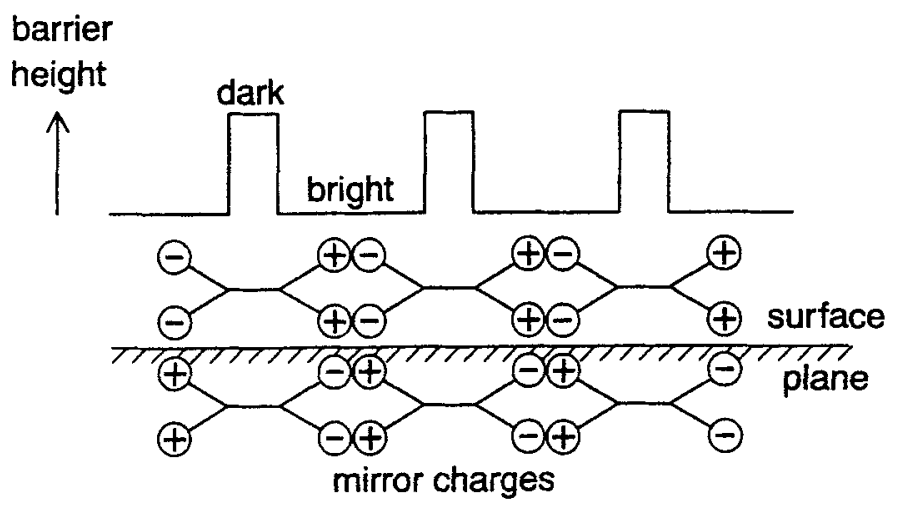

Fig. 5 Due to the induced mirror charges the dipole moments of the polar polymer are oriented parallel to the conducting graphite surface. This leeds to an alternating structure of high and low polarizability. The thin dark lines in the STM images are the polymer skeleton (low polarizability) and the bright stripes are the polar sidegroups of two adjacent chains (high polarizability).

ability in the domain walls, they appear dark in the STM image, is compatible with a reduced polarizability of the chains parallel to the dipolar axis and to the potential difference in dipole direction, both increasing the effective barrier height. The observed width of the domain walls of $5-10 \AA$ is in good agreement with the calculated width of domain walls [5]. In the bright granular stripes the underlying graphite lattice is always visible at larger magnification. We believe therefore that the image contrast results from the modulation of the graphite barrier height by the polymer monolayer. In this case of an insulating molecular system tunneling occurs through the molecule. In contrast, for a semiconducting dye molecule such as PTCDA [6] the imaging is determined by tunneling from the molecule to the scanning tip.

In the following we will compare our results with the two models $[4,5]$ of kink motion mentioned above. Both models are based on a thermal generation of kinks. In the model of Dvey-Aharon the height of the potential wall between two $60^{\circ}$ domains is about $7 k T_{0}$ or $180 \mathrm{meV}$, but the domain walls are assumed to be parallel to the chains. Pertsev finds that the activation energy for domain walls normal to the chains is lower than for domain walls parallel to the chains, but without giving numerical data under zero field conditions. From our preliminary experiments we obtain an activation energy of about 200-300 meV from the Arrhenius diagram. Apart from the fact that we have a two dimensional arrangement of the chains and therefore the possibility of only $180^{\circ}$ domains the higher value seems more reasonable. The main difference between the two models concerns the direction of the domain walls. Our experiments clearly show domain walls at right angles to the chain direction. Therefore the model of collective kink motion as proposed by Pertsev appears to be more appropriate for the description of our results on epitaxial PVDF monolayers.

The fact that we can observe domain wall motions on a time scale of $100 \mathrm{~ms}$ at room temperature in small crystallites is in strong contradiction to an intrinsic hard ferroelectric behavior of PVDF. This implies, under the assumption that the two-dimensional system investigated in our experiments shows a similar dynamic behavior as bulk PVDF, that there must be an additional mechanism as charge trapping [3] at the crystallite surfaces, to stabilize the domain walls in poled PVDF films. 
In summary, we have demonstrated that it is possible after a special pretreatment of graphite to prepare epitaxial P(VDF/TrFE) monolayers from solution. Due to the induced mirror charges the dipole moments of the polar polymer are parallel to the surface plane and the lattice constant of the monolayers is compressed perpendicular to the chain direction by $13 \%$ compared to the bulk structure being in registry with every second next carbon row of the graphite surface. With our Video-STM we are able to observe thermal domain wall motion as well as thermal grain boundary motion in real time with a time resolution of $100 \mathrm{~ms}$. The domain wall velocity seems to depend exponentially on the temperature as expected for a thermally activated motion. From the two theoretical models under discussion which tried to describe the mechanism of domain wall motion, the model of collective kink motion by Pertsev [5] corresponds better to our results. The observed kink motion at room temperature is furthermore in agreement with the requirement of charge trapping at crystallite surfaces for the explanation of the hard ferroelectricity of PVDF.

The authors would like to thank K. Laßmann and W. Glatz for helpful discussions. This research was supported by the Sonderforschungsbereich 329 .

\section{References}

[1] H. Kawai, Jap. J. Appl. Phys. 8 (1968) 975

[2] E. Bihler, G. Neumann, G. Eberle, W. Eisenmenger, IEEE 1990 Annual Report Conf. Elect. Insul. Dielect. Phen. 140

[3] W. Eisenmenger, M. Haardt, Sol. State Comm. 41 (1982) 917

[4] H. Dvey-Aharon, T. J. Sluckin, P. L. Taylor, A. J. Hopfinger, Phys. Rev. B21 (1980) 3700

[5] N. A. Pertsev, A. G. Zembil'gotov, Sov. Phys. Solid State 33 (1991) 165

[6] Ch. Ludwig, B. Gompf, W. Glatz, J. Petersen, W. Eisenmenger, M. Möbus, U. Zimmermann, N. Karl, Z. Phys. B Condensed Matter 86 (1992) 397

[7] H. Fuchs, S. Akari, K. Dransfeld, Z. Phys.-Condensed Matter 80 (1990) 389

[8] M. Specht, F. Ohnesorge, W.M. Heckl, Surf. Sci. Lett. 257 (1991) L653

[9] H. Birk, J. Glatz-Reichenbach, Li-Jie, E. Schreck, K. Dransfeld, J. Vac. Sci. Technol. B9 (1991) 1162

[10] J. P. Rabe, S. Buchholz, Phys. Rev. Lett. 66 (1991) 2096

[11] I. Fujiwara, C. Ishimoto, J. Seto, J. Vac. Sci. Technol. B9 (1991) 1148

[12] R. LeBihan, M. Maussion, J. Physique 33 (1972) C2-215

[13] Peng ju lin, L.A. Bursill, Philos. Mag. A 48 (1983) 251

[14] F. Saurenbach, B.D. Terris, Appl. Phys. Lett. 56 (1990) 1703

[15] M.G. Broadhurst, G.T. Davis, in: Electrets, G.M. Sessler (ed.), Springer, 1987, p. 285

[16] J. B. Lando, W.W. Doll, J. Macromol. Sci.-Phys. B 2 (1968) 205

[17] J.K. Spong, H.A. Mizes, L. J. LaComb, M.M. Dovek, J.E. Frommer, J.S. Foster, Nature 338 (1989) 137 